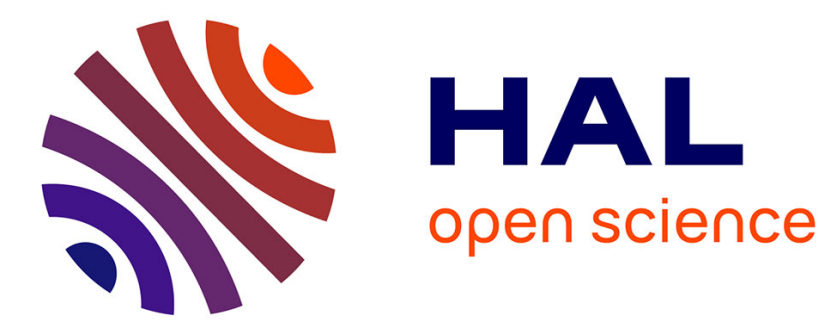

\title{
CFO sensitivity and efficient joint estimation for the short filter multicarrier system FBMC-PAM
}

\author{
Davide Mattera, Mario Tanda, Maurice Bellanger
}

\section{To cite this version:}

Davide Mattera, Mario Tanda, Maurice Bellanger. CFO sensitivity and efficient joint estimation for the short filter multicarrier system FBMC-PAM. Signal Processing, 2019, 164, pp.10 - 19. 10.1016/j.sigpro.2019.05.031 . hal-02476575

\section{HAL Id: hal-02476575 \\ https://hal.science/hal-02476575}

Submitted on 27 Nov 2020

HAL is a multi-disciplinary open access archive for the deposit and dissemination of scientific research documents, whether they are published or not. The documents may come from teaching and research institutions in France or abroad, or from public or private research centers.
L'archive ouverte pluridisciplinaire HAL, est destinée au dépôt et à la diffusion de documents scientifiques de niveau recherche, publiés ou non, émanant des établissements d'enseignement et de recherche français ou étrangers, des laboratoires publics ou privés. 


\title{
CFO sensitivity and efficient joint estimation for the short filter multicarrier system FBMC-PAM
}

\author{
Davide Mattera $^{\mathrm{a}}$, Mario Tanda ${ }^{\mathrm{a}, *}$, Maurice Bellanger ${ }^{\mathrm{b}}$ \\ ${ }^{a}$ Dipartimento di Ingegneria Elettrica e delle Tecnologie dell'Informazione, \\ Università degli Studi di Napoli Federico II, via Claudio 21, I-80125 Napoli, Italy. \\ ${ }^{b}$ CNAM-Electronique, 292 rue Saint-Martin, 75141 Paris cedex 03, France.
}

\begin{abstract}
Sensitivity to carrier frequency offset, due to oscillator inaccuracy and terminal mobility, is a key issue that modern multicarrier systems have to face. In that respect, FBMC-PAM holds a specific position due to its short prototype filter: the overlapping factor is $\mathrm{K}=2$ and the width of the main lobe of its frequency response is 3 times the sub-carrier spacing, while OFDM and FBMC-OQAM schemes have frequency response whose main lobe width is 2 times the sub-carrier spacing. As a consequence, it is shown in the present paper that FBMC-PAM outperforms other multicarrier techniques in terms of CFO sensitivity. In order to best exploit this property in burst transmission, an efficient and accurate approach for joint symbol timing and CFO estimation is proposed, based on a specific preamble. A theoretical in-depth analysis of the scheme is provided as well as performance validation in multipath channel through simulations.
\end{abstract}

Keywords: FBMC, OFDM, PAM, lapped transform, SIR

\footnotetext{
*Corresponding author. Tel. +39-081-768-3791. Fax +39-081-768-5925.

Email addresses: mattera@unina. it (Davide Mattera), tanda@unina . it (Mario Tanda), bellang@cnam.fr (Maurice Bellanger)
} 


\section{Introduction}

Filter bank multicarrier (FBMC) techniques are proposed as alternatives to the current orthogonal frequency division multiplexing (OFDM) schemes for emerging applications fields such as machine type communications (MTC) or cognitive radio [1]. A critical parameter in these systems is the prototype filter which controls performance and a number of operational aspects. Long filters yielding high performance, particularly in terms of out-of-band attenuation and user coexistence, have been proposed. However, in the scenarios requiring reduced system latency or robustness to time-varying channels, short prototype filters are preferred. A number of approaches based on prototype filters with overlapping factor $\mathrm{K}=2$, i.e. only 2 adjacent multicarrier symbols overlap in time, have been proposed $[2,3]$. An additional important benefit of these techniques is a reduction of implementation complexity. However, when offset-quadrature amplitude modulation (OQAM) is combined with short filters, the level of performance achieved is limited, making the usefulness of such schemes questionable. That is why FBMC-PAM has been introduced [4]. The scheme is based on pulse amplitude modulation (PAM) combined with a sine prototype filter with overlapping factor $\mathrm{K}=2$ and it achieves full orthogonality, like OFDM. Regarding mobile communications and the issue of carrier frequency offset (CFO) sensitivity the sine prototype filter exhibits a key characteristic, namely the width of the main lobe of its frequency response is 1.5 times that of OFDM and FBMC-OQAM schemes. The consequence of this enlarged main lobe is that FBMC-PAM outperforms other multicarrier techniques in sensitivity to CFO. Now, in order to benefit from this intrinsic advantage in mobile communications, accurate yet efficient estimation and synchronization techniques for burst transmission must be implemented. Such techniques have been developed for data-aided synchronization of FBMC-OQAM systems [5-7]. They can be adapted to FBMC-PAM and 
optimized in the specific context. Here, preamble-based joint symbol-timing and CFO estimation is retained and a synchronization algorithm exploiting the least squares method is derived in AWGN channel and analyzed in standard multipath channels. The considered preamble can be easily generated, allows to maintain the appealing spectral-shaping properties of the FBMC-PAM signal and assures interesting performance in multipath channels.

The organization of the paper is as follows. In Section 2, the standard FBMCPAM system is recalled while in Secion 3 its CFO sensitivity is analyzed. In Section 4 the proposed preamble-based symbol timing and CFO estimation algorithm is derived. The Cramér-Rao bound (CRB) on CFO and phase offset estimation for the problem at hand is derived in Section 5. Numerical results are reported in Section 6 and conclusions are drawn in the final Section.

Notation: $j \triangleq \sqrt{-1}$, superscript $(\cdot)^{*}$ and $(\cdot)^{T}$ denote the complex conjugation, and the transpose, respectively, $\mathfrak{R}[\cdot]$ the real part, $\delta[k]$ the Kronecker delta, $|\cdot|$ the absolute value, $\angle[\cdot]$ the argument of a complex number in $[-\pi, \pi), E[\cdot]$ denotes statistical expectation, and $\bmod _{M}(\ell) \triangleq \ell-q M$ with $q$ such that $\bmod _{M}(\ell) \in$ $\{0,1, \ldots, M-1\}$.

\section{System Model}

Let us consider an FBMC-PAM system [4] with $2 M$ subcarriers. The received signal in AWGN channel, in the presence of a timing offset $\tau$, a carrier-frequency offset $\Delta f$, a carrier phase offset $\phi$ and an attenuation $\gamma$ can be written as

$$
r(t)=\gamma s(t-\tau) e^{j 2 \pi \Delta f t} e^{j \phi}+n(t)
$$

where $s(t)$ is the transmitted FBMC-PAM signal and $n(t)$ denotes the circular complex white Gaussian noise with two-sided power spectral density $2 N_{o}$. The 
FBMC-PAM signal $s(t)$ is equal to

$$
s(t)=\sum_{i=0}^{N_{b}+N_{s}-1} \sum_{k=0}^{2 M-1} d_{k}(i) e^{j \frac{\pi}{T}\left(k+\frac{1}{2}\right)\left(t-i T+\frac{T}{2}\right)} h(t-i T)
$$

where $2 T$ is the FBMC-PAM symbol duration, $N_{b}$ is the number of training symbols, $N_{s}$ is the number of payload symbols, $d_{k}(i)$ denotes the real data symbol transmitted on the $k$ th subcarrier during the $i$ th FBMC-PAM symbol, and $h(t)$ is the real pulse-shaping filter.

In the following we assume that the received signal $r(t)$ is filtered with an ideal low-pass filter with a bandwidth of $1 / T_{s}$, where $T_{s}$ denotes the sampling period. Note that, the FBMC-PAM symbol duration is equal to $2 T=2 M T_{s}$.

The discrete-time low-pass version of the received signal can be written as

$$
r[l]=\gamma s[l-\theta] e^{j \frac{2 \pi}{2 M} \epsilon l} e^{j \phi}+v[l]
$$

where $\theta=\tau / T_{s}$ is the normalized delay (assumed to be an integer), $\epsilon=\Delta f 2 T$ is the CFO normalized to subcarrier spacing $\delta f=1 /(2 T)$ and, moreover, $v[l]$ is a discrete-time zero-mean AWGN process with autocorrelation function

$$
R_{v}[m]=E\left[v[l] v^{*}[l-m]\right]=\frac{2 N_{o}}{T_{s}} \delta[m] .
$$

In (3) the signal of interest can be written as

$$
s[l]=\sum_{i=0}^{N_{b}+N_{s}-1} \sum_{k=0}^{2 M-1} d_{k}[i] \mathcal{T}_{c}[k, l-i M]
$$

where

$$
\mathcal{T}_{c}[k, l] \triangleq h[l] e^{j \frac{\pi}{M}\left(k+\frac{1}{2}\right)\left(l+\frac{1}{2}+\frac{M}{2}\right)} .
$$

In (6) the real prototype filter $h[l]$ (equal to zero for $l \notin \mathcal{K}_{2 M} \triangleq\{0,1, \ldots, 2 M-1\}$ ) satisfies the following conditions

$$
\begin{array}{r}
h[M+l]=h[M-l-1] \quad \forall l, \\
\sum_{m=-\infty}^{+\infty} h[l+m M] h[l+m M+2 r M]=\delta[r] \quad \forall l, r .
\end{array}
$$


In particular, (7) is a symmetry condition, while (8) represents a condition on the zeros, at frequencies $F_{k}=k / M$, of the Fourier transform of the generator of the periodic signal in the left hand side. Since the prototype filter $h[l]$ is equal to zero for $n \notin \mathcal{K}_{2 M}$, condition (8) for $r=0$ simply becomes

$$
h^{2}[l]+h^{2}[l+M]=1, \forall l .
$$

Note that conditions (7) and (8) imply the orthogonality condition

$$
\frac{1}{M} \mathfrak{R}\left\{\sum_{l=0}^{2 M-1} \mathcal{T}_{c}[k, l] \mathcal{T}_{c}^{*}[m, l-p M]\right\}=\delta[m-k] \delta[p] .
$$

In this case, it follows from [4] that in AWGN channel $(\epsilon=\theta=0$ in (3)) the optimum (in the maximum likelihood sense) decision variable for estimating statistically independent information symbols can be written as

$$
\hat{d}_{m}[i] \triangleq \frac{1}{M} \mathfrak{R}\left\{e^{-j \phi} z_{m}(i)\right\}
$$

where

$$
z_{m}(i) \triangleq \sum_{l=0}^{2 M-1} r[i M+l] \mathcal{T}_{c}^{*}[m, l]
$$

since conditions (7) and (8) assure the absence of intersymbol interference and intercarrier interference. Specifically, the symmetry condition in (7) assures, in the decisions on the data transmitted on the $m$-th subcarrier, the absence of intersymbol interference and, moreover, the absence of intercarrier interference from subcarriers whose index $k$ is such that $\Delta=\bmod _{2 M}(k-m)$ is an odd number. In addition, condition (8) assures the absence of intercarrier interference from the subcarriers whose index $k$ is such that $\Delta=\bmod _{2 M}(k-m)$ is an even number.

In the following it is considered the prototype filter

$$
h[l]=\sin \left[\frac{\pi}{2 M}\left(l+\frac{1}{2}\right)\right] \quad l \in \mathcal{K}_{2 M}, \quad h[l]=0 \quad l \notin \mathcal{K}_{2 M} .
$$

This prototype filter satisfies conditions (7) and (8), exhibits good spectral properties and simplifies the receiver as shown in [4]. 
Let us observe that accounting for (6), (12) and (13) it follows that

$$
\begin{gathered}
z_{m}(i)=\sum_{l=0}^{2 M-1} r[i M+l] h[l] e^{-j \frac{\pi}{M}\left(m+\frac{1}{2}\right)\left(l+\frac{1}{2}+\frac{M}{2}\right)} \\
=e^{-j \frac{\pi}{M}\left(m+\frac{1}{2}\right)\left(\frac{1}{2}+\frac{M}{2}\right)}\left[a^{*} R_{m}(i)+a R_{m+1}(i)\right]
\end{gathered}
$$

where

$$
a \triangleq-\frac{e^{-j \frac{\pi}{4 M}}}{2 j}
$$

and

$$
R_{m}(i) \triangleq \sum_{l=0}^{2 M-1} r[i M+l] e^{-j \frac{2 \pi}{2 M} l m} .
$$

Thus, an efficient evaluation of the term $z_{m}(i)$ in (14) requires the evaluation of an FFT over $2 M$ points. In order to boost the performance of the frequency domain channel equalizer, the 2M-FFT can be replaced by a 4M-FFT followed by decimation. The scheme is denominated FBMC-PAM-4M and details are available in [4].

\section{Carrier frequency offset sensitivity of the FBMC-PAM modulation scheme}

In this section we evaluate the sensitivity of the FBMC-PAM system to the residual CFO. In particular, we obtain an approximate expression for the SIR, that is the ratio between the power of the useful term and the power of the interference present in the decision variable due to the CFO.

Let us observe that the decision variable in (11) on the datum transmitted on the $m$-th subcarrier in the $i$-th multicarrier symbol, taking into account (12) and (14), can be written as

$$
\hat{d}_{m}[i]=\frac{1}{M} \mathfrak{R}\left\{e^{-j \phi} e^{-j \frac{\pi}{M}\left(m+\frac{1}{2}\right)\left(\frac{1}{2}+\frac{M}{2}\right)}\left[a^{*} R_{m}(i)+a R_{m+1}(i)\right]\right\} .
$$

To evaluate the sensitivity of the FBMC-PAM to a normalized CFO $\epsilon$, let us consider the discrete-time received signal in (3) in the absence of noise for $\theta=0$ and, 
without loss of generality, for $\gamma=1$. In this case the $m$-th value of the DFT in (17) depends also on $\epsilon$. Taking into account its expression derived in Appendix A, it follows that

$$
\begin{gathered}
\hat{d}_{m}[i]=\frac{1}{M} \mathfrak{R}\left\{e^{-j \pi \epsilon i} e^{-j \frac{\pi}{2} \epsilon\left(2-\frac{1}{M}\right)} e^{-j \frac{\pi}{M}\left(m+\frac{1}{2}\right)\left(\frac{1}{2}+\frac{M}{2}\right)}\left[a^{*} R_{m}(i, \epsilon)+a R_{m+1}(i, \epsilon)\right]\right\} \\
=\frac{1}{4 M} \sin \left(\frac{\pi}{2} \epsilon\right)(-1)^{i} \sum_{k=0}^{2 M-1} d_{k}[i-1]\left\{D_{2 M}^{m-k-1}(\epsilon)+D_{2 M}^{m-k+1}(\epsilon)\right\} \\
+\frac{1}{4 M} \sum_{k=0}^{2 M-1} d_{k}[i]\left\{2 D_{4 M}^{2(m-k)}(2 \epsilon)+D_{4 M}^{2(m-k-1)}(2 \epsilon)+D_{4 M}^{2(m-k+1)}(2 \epsilon)\right\} \cos \left(\frac{3 \pi}{2}(k-m)\right) \\
+\frac{1}{4 M} \sin \left(\frac{\pi}{2} \epsilon\right) \sum_{k=0}^{2 M-1}(-1)^{k} d_{k}[i+1]\left\{D_{2 M}^{m-k-1}(\epsilon)+D_{2 M}^{m-k+1}(\epsilon)\right\}
\end{gathered}
$$

where

$$
D_{M}^{p}(x) \triangleq \frac{\sin \left[\frac{\pi}{2}(p-x)\right]}{\sin \left[\frac{\pi}{M}(p-x)\right]} .
$$

Note that in (18) has been used the complex gain $w=e^{-j \pi \epsilon i} e^{-j \frac{\pi}{2} \epsilon\left(2-\frac{1}{M}\right)}$ to compensate for the phase offset and the CFO (constant on each subacarrier) accumulated up to the considered multicarrier symbol. In this way, only the effect of the residual inter-channel interference (ICI) and inter-symbol interference (ISI) due to the undesired factor $e^{j \frac{\pi}{M} \epsilon l}$ in the sums is accounted for (see e.g., [8]). The SIR $\rho(\epsilon)$ is given by

$$
\rho(\epsilon)=\frac{P_{u}(\epsilon)}{P_{I S I}(\epsilon)+P_{I C I}(\epsilon)}
$$

where (see (18))

$$
P_{u}(\epsilon)=\left[2 D_{4 M}^{0}(2 \epsilon)+D_{4 M}^{-2}(2 \epsilon)+D_{4 M}^{2}(2 \epsilon)\right]^{2}
$$

is the power of the useful term while

$$
P_{I S I}(\epsilon)=2\left[\sin \left(\frac{\pi}{2} \epsilon\right)\right]^{2} \sum_{k=0}^{2 M-1}\left[D_{2 M}^{m-k+1}(\epsilon)+D_{2 M}^{m-k-1}(\epsilon)\right]^{2}
$$


and

$$
P_{I C I}(\epsilon)=\sum_{k=0, k \neq m}^{2 M-1} \frac{\left[1+(-1)^{k-m}\right]}{2}\left[2 D_{4 M}^{2(m-k)}(2 \epsilon)+D_{4 M}^{2(m-k-1)}(2 \epsilon)+D_{4 M}^{2(m-k+1)}(2 \epsilon)\right]^{2}
$$

are the power of ISI and ICI contribution, respectively. In Appendix B it is shown that, for $|\epsilon| \ll 1$ and $M \gg 1$, the power of the useful term in (21) can be approximated as

$$
P_{u}(\epsilon) \simeq 16 M^{2}
$$

the ISI power can be approximated as

$$
P_{I S I}(\epsilon) \simeq \pi^{2} \epsilon^{2} M^{2}\left(\frac{5}{3}+\frac{4}{\pi^{2}}\right)
$$

while the ICI power can be approximated as

$$
P_{I C I}(\epsilon) \simeq \pi^{2} \epsilon^{2} M^{2} 12\left(\frac{10-\pi^{2}}{\pi^{2}}\right)
$$

Thus, for $|\epsilon| \ll 1$ and $M \gg 1$, the SIR can be approximated as

$$
\rho(\epsilon) \simeq \frac{1}{\pi^{2} \epsilon^{2}\left[\frac{5}{48}+\frac{1+3\left(10-\pi^{2}\right)}{4 \pi^{2}}\right]} .
$$

Note that, for a given value of $\epsilon$, the power of the interference is nearly equal to that of the ISI contribution since from (25) and (26) it follows that $P_{I C I}(\epsilon) \simeq$ $0.076 P_{I S I}(\epsilon)$.

It is of interest to compare the derived SIR expression for FBMC-PAM systems with the approximate expression for OFDM systems obtained in [8]

$$
\rho^{O F D M}(\epsilon) \simeq \frac{1}{\frac{\pi^{2} \epsilon^{2}}{3}}
$$

In Figure 1 are reported the approximate SIR in (27) (labeled as FBMC-PAMA) and the exact SIR (labeled as FBMC-PAM) obtained by using (21), (22) and (23) in (20). Moreover, in the figure the exact SIR for an OFDM system (labeled 
as OFDM) and its approximate value given by (28) (labeled as OFDM-A) are also reported. The results show that, in the considered range, of values of $\epsilon$, the obtained approximate expressions provide results nearly coincident with the exact resuts. In particular, the gain $\alpha \triangleq \frac{\rho(\epsilon)}{\rho^{O F D M}(\epsilon)}$ of FBMC-PAM with respect to OFDM in terms of SIR, expressed in $\mathrm{dB}$, is nearly equal to $3.8 \mathrm{~dB}$ (the actual value of the ratio $\alpha$ obtained by using the exact SIR expressions is nearly equal to $4 \mathrm{~dB}$ ). In addition, let us observe that the approximate SIR expression in (27) has the form $\rho(\epsilon) \simeq 1 /\left(k_{1} \epsilon^{2}\right)$ obtained in [8] for offset-QAM multicarrier systems. Since for FBMC-PAM $k_{1} \simeq 1.38$ while for OFDM, SR-RC, SR-Nyquist, IOTA and Hermite designs, the values of $k_{1}$ are (see [8]) 3.29, 2.3, 1.88, 1.54, and 1.55, respectively, it follows that FBMC-PAM is superior to these systems provided that they are subject to the same CFO. This is obtained by choosing a value of $\mathrm{M}$ for FBMC-PAM equal to half of that of the other considered systems. In fact, in this case the subcarrier spacing is the same and also the frequency offset for a given normalized CFO $\epsilon$.

\section{Joint symbol timing and carrier frequency offset LS estimator}

Let us consider the case where $N_{b}=2$ in (5), and, moreover, $d_{k}[1]=0, \forall k$ and $d_{k}[0]=0$, for even $k$, that is, the preamble is obtained by transmitting real data only on odd subcarriers in the first multicarrier symbol of the burst. In this case, the samples of the preamble in (5) can be written as

$$
p[l]=h[l] z[l], \quad l \in \mathcal{K}_{2 M}
$$

where

$$
z[l] \triangleq \sum_{k=0}^{2 M-1} d_{k}[0] e^{j \frac{\pi}{M}\left(k+\frac{1}{2}\right)\left(l+\frac{1}{2}+\frac{M}{2}\right)} .
$$


Let us observe that (for $l \in\{0,1, \ldots, M-1\}$ )

$$
z[l+M]=\sum_{k=0}^{2 M-1} d_{k}[0] e^{j \frac{\pi}{M}\left(k+\frac{1}{2}\right)\left(l+M+\frac{1}{2}+\frac{M}{2}\right)}=\sum_{k=0}^{2 M-1} d_{k}[0] e^{j \frac{\pi}{M}\left(k+\frac{1}{2}\right)\left(l+\frac{1}{2}+\frac{M}{2}\right)} e^{j \frac{\pi}{2}}(-1)^{k} .
$$

Since it is assumed that only odd subcarriers are modulated (i.e., $d_{k}[0]=0$, for even $k$ ) it immediately follows that the samples of the signal $z[l]$ in (30) satisfy (for $l \in\{0,1, \ldots, M-1\}$ ) the following property:

$$
z[l+M]=-j z[l]
$$

Taking into account (29) and (3) it follows that in the absence of noise and for $l \in\{0,1, \ldots, M-1\}$

$$
r[l+\theta]=\gamma h[l] z[l] e^{j \frac{2 \pi}{2 M} \epsilon(l+\theta)} e^{j \phi}
$$

and, moreover,

$$
r[l+M+\theta]=\gamma h[l+M] z[l+M] e^{j \frac{2 \pi}{2 M} \epsilon(l+M+\theta)} e^{j \phi} .
$$

Therefore, taking into account the property in (32), from (33) and (34) and for $l \in\{0,1, \ldots, M-1\}$, in the absence of noise we can write

$$
r[l+M+\theta] h[l]=-j e^{j \pi \epsilon} r[l+\theta] h[l+M] .
$$

Thus, from (35) it follows that the joint estimate of the timing-offset $\theta$ and of the normalized CFO $\epsilon$ according to the LS approach is obtained as follows

$$
(\hat{\theta}, \hat{\epsilon})=\arg \min _{\tilde{\theta}, \tilde{\epsilon}}\{U(\tilde{\theta}, \tilde{\epsilon})\}
$$

where

$$
U(\tilde{\theta}, \tilde{\epsilon}) \triangleq \sum_{l=0}^{M-1}\left|r[l+M+\tilde{\theta}] h[l]+j r[l+\tilde{\theta}] h[l+M] e^{j \pi \tilde{\epsilon}}\right|^{2}
$$

and $\tilde{\theta}$ and $\tilde{\epsilon}$ are trial values for timing-offset and CFO, respectively. 
It can be easily shown that the CFO estimate is expressed in closed form by

$$
\hat{\epsilon}=\frac{1}{\pi} \angle S(\hat{\theta})+\frac{1}{2}
$$

where

$$
S(\theta) \triangleq \sum_{l=0}^{M-1} h[l] h[l+M] r^{*}[l+\theta] r[l+M+\theta] .
$$

In particular, taking into account the considered prototype filter in (13) it immediately follows that

$$
S(\theta)=\frac{1}{2} \sum_{l=0}^{M-1} \sin \left[\frac{\pi}{2 M}(2 l+1)\right] r^{*}[l+\theta] r[l+M+\theta] .
$$

By substituting the CFO estimate (38) in (36), it follows that the timing-offset estimate requires only a one-dimensional search and is given by

$$
\hat{\theta}=\arg \max _{\tilde{\theta}}\left\{|S(\tilde{\theta})|-\frac{1}{2} W(\tilde{\theta})\right\}
$$

where $S(\theta)$ is defined in (39) and

$$
W(\theta) \triangleq \sum_{l=0}^{M-1}\left[|r[l+M+\tilde{\theta}]|^{2} h^{2}[l]+|r[l+\tilde{\theta}]|^{2} h^{2}[l+M]\right] .
$$

To simplify the threshold setting (see [9] and references therein) in the next section the following modified version of (41) is considered:

$$
\hat{\theta}=\arg \max _{\tilde{\theta}}\left\{\frac{2|S(\tilde{\theta})|}{W(\tilde{\theta})}\right\} .
$$

Note that the proposed CFO estimator in (38) assures unambiguous estimates provided that $\epsilon \in[-0.5,1.5)$ while the proposed symbol timing estimator in (43) assures unambiguous estimates in the whole range $\theta \in\{0,2 M-1\}$. To enlarge the CFO estimation range, a preamble symbol consisting of $L>2$ (weighted) identical parts can be exploited (see e.g., $[10,11]$ for the OFDM case). This can be obtained by transmitting the real data on the subcarriers whose index is an integer 
multiple of $L$ and setting zero on the remaining subcarriers. In fact, in this case the CFO estimation range is multiplied by $L$. Moreover, although the proposed joint preamble-based symbol-timing and CFO estimator has been derived by exploiting the LS approach with reference to an additive noise channel model, in the following its performance is assessed in standard multipath channels.

Figure 2 shows the behaviour, in a single run, of the proposed preamble-based symbol timing statistics in AWGN as a function of $\tilde{\theta}$ for $E_{b} / N_{o}=10 \mathrm{~dB}$ and $M=512$. The proposed estimator presents a peak at the actual value of the delay $\theta=M$.

\section{Derivation of CRB}

In this section we assume that the symbol timing in (3) is perfectly known and derive the expression of the $\mathrm{CRB}$ for joint $\mathrm{CFO}$ and phase estimation. Let $\mathbf{r}$ be the observation vector, $\mathbf{v}=[\epsilon, \phi]^{T}$ the parameters to be estimated, the $(i, l)$ th entry of the Fisher Information Matrix (FIM) is equal to

$$
[\mathbf{F}]_{(i, l)}=-\mathrm{E}_{\mathbf{r}}\left[\frac{\partial^{2} \ln p_{\mathbf{r}}(\mathbf{r} \mid \mathbf{v})}{\partial[\mathbf{v}]_{i} \partial[\mathbf{v}]_{l}}\right]
$$

where $E_{\mathbf{r}}$ is the expectation with respect to $\mathbf{r}$ and (up to irrelevant additive factors)

$$
\ln p_{\mathbf{r}}(\mathbf{r} \mid \mathbf{v})=\frac{\gamma T_{s}}{N_{o}} \sum_{l=0}^{2 M-1} \mathfrak{R}\left\{r^{*}[l] p[l] e^{j \frac{2 \pi}{2 M} l \epsilon} e^{j \phi}\right\} .
$$

It results that

$$
\begin{gathered}
\frac{\partial^{2} \ln p_{\mathbf{r}}(\mathbf{r} \mid \mathbf{v})}{\partial \epsilon^{2}}=-\frac{\gamma T_{s}}{N_{o}}\left(\frac{\pi}{M}\right)^{2} \sum_{l=0}^{2 M-1} \mathfrak{R}\left\{l^{2} r^{*}[l] p[l] e^{j \frac{2 \pi}{2 M} l \epsilon} e^{j \phi}\right\}, \\
\frac{\partial^{2} \ln p_{\mathbf{r}}(\mathbf{r} \mid \mathbf{v})}{\partial \epsilon \partial \phi}=\frac{\partial^{2} \ln p_{\mathbf{r}}(\mathbf{r} \mid \mathbf{v})}{\partial \phi \partial \epsilon}=-\frac{\gamma T_{s}}{N_{o}} \frac{\pi}{M} \sum_{l=0}^{2 M-1} \mathfrak{R}\left\{l r^{*}[l] p[l] e^{j \frac{2 \pi}{2 M} l \epsilon} e^{j \phi}\right\},
\end{gathered}
$$

and, moreover

$$
\frac{\partial^{2} \ln p_{\mathbf{r}}(\mathbf{r} \mid \mathbf{v})}{\partial \phi^{2}}=-\frac{\gamma T_{s}}{N_{o}} \sum_{l=0}^{2 M-1} \mathfrak{R}\left\{r^{*}[l] p[l] e^{j \frac{2 \pi}{2 M} l \epsilon} e^{j \phi}\right\} .
$$


Taking into account (46), (47), (48) and (44) we obtain

$$
\begin{gathered}
{[\mathbf{F}]_{(1,1)}=\frac{\gamma^{2} T_{s}}{N_{o}}\left(\frac{\pi}{M}\right)^{2} \sum_{l=0}^{2 M-1} l^{2}|p[l]|^{2},} \\
{[\mathbf{F}]_{(2,1)}=[\mathbf{F}]_{(1,2)}=\frac{\gamma^{2} T_{s}}{N_{o}} \frac{\pi}{M} \sum_{l=0}^{2 M-1} l|p[l]|^{2},}
\end{gathered}
$$

and

$$
[\mathbf{F}]_{(2,2)}=\frac{\gamma^{2} T_{s}}{N_{o}} \sum_{l=0}^{2 M-1}|p[l]|^{2} .
$$

The CRB for normalized CFO and for phase is given by the corresponding diagonal element of inverse of FIM, that is

$$
C R B(\epsilon)=\left[\mathbf{F}^{-1}\right]_{(1,1)}=\frac{1}{\left(\frac{\pi}{M}\right)^{2} \frac{\gamma^{2} T_{s}}{N_{o}} \sum_{l=0}^{2 M-1}|p[l]|^{2} \beta}
$$

and

$$
C R B(\phi)=\left[\mathbf{F}^{-\mathbf{1}}\right]_{(2,2)}=\frac{\alpha}{\frac{\gamma^{2} T_{s}}{N_{o}} \sum_{l=0}^{2 M-1}|p[l]|^{2} \beta}
$$

where

$$
\alpha \triangleq \frac{\sum_{l=0}^{2 M-1} l^{2}|p[l]|^{2}}{\sum_{l=0}^{2 M-1}|p[l]|^{2}}
$$

and

$$
\beta \triangleq \frac{\sum_{l=0}^{2 M-1} l^{2}|p[l]|^{2}}{\sum_{l=0}^{2 M-1}|p[l]|^{2}}-\left(\frac{\sum_{l=0}^{2 M-1} l|p[l]|^{2}}{\sum_{l=0}^{2 M-1}|p[l]|^{2}}\right)^{2} .
$$

Let us observe that the parameters $\alpha$ and $\beta$, in (54) and (55), respectively, do not depend on the preamble energy but only on its shape. 


\section{Simulation results}

In this section the performance of the proposed preamble-based joint symbol timing and $\mathrm{CFO}$ estimator is assessed via computer simulations. The simulation results are obtained under the following conditions:

1. the total number of subcarriers for the considered FBMC-PAM system is $2 M=1024$, the number of active subcarriers is 912 and, moreover, the transmitted data belong to a 2-PAM constellation;

2. the total number of subcarriers for the considered OFDM system is $M=$ 1024 , the number of active subcarriers is 840 , and, moreover, the transmitted data belong to a 4-QAM constellation;

3. the considered multipath channel model Extended Vehicular A (EVA) [12] has the following power/delay profile: relative power (in $\mathrm{dB}$ ) equal to $\left[\begin{array}{lllllllll}0 & -1.5 & -1.4 & -3.6 & -0.6 & -9.1 & -7 & -12 & -16.9\end{array}\right]^{T}$ and delay (in discrete samples) $\left[\begin{array}{lllllllll}0 & 1 & 3 & 6 & 7 & 14 & 22 & 35 & 50\end{array}\right]^{T}$. Moreover, the considered multipath channel model Extended Typical Urban (ETU) [12] has the following power/delay profile: relative power (expressed in $\mathrm{dB}$ ) equal to $\left[\begin{array}{llllllllll}-1 & -1 & -1 & 0 & 0 & 0 & -3 & -5 & -7\end{array}\right]^{T}$ and delay (expressed in discrete samples) equal to $\left[\begin{array}{lllllllll}0 & 1 & 2 & 4 & 5 & 10 & 32 & 46 & 100\end{array}\right]^{T}$;

4. in each run the actual value of the symbol timing is a realization of a random variable uniformly distributed in the set $\{0,2 M-1\}$ while each CFO value is a realization of a random variable uniformly distributed in $[-0.4,1.4)$;

5. the normalized root-mean-square error (RMSE) values are obtained by averaging over $10^{4}$ independent channel realizations;

6. the BER values are obtained by averaging over bursts of 20 payload symbols received in $10^{4}$ independent channel realizations;

7. each channel realization remains constant in the whole burst, and, moreover, the equalizer has a perfect knowledge of the channel with the residual timing 
offset.

Figure 3 shows the RMSE normalized to $M$ of the proposed symbol timing estimator as a function of $E_{b} / N_{o}$ in AWGN and in multipath channels EVA and ETU. The results show that a performance degradation is observed in multipath channel with respect to that obtained in AWGN channel, in particular a floor is observed. However, the timing estimate is quite accurate also in the highly frequency selective ETU channel provided that $E_{b} / N_{o} \geq 5 \mathrm{~dB}$. Note that in this range of values of $E_{b} / N_{o}$ the RMSE value of the symbol timing estimate is nearly equal to $3 \%$ of the FBMC-PAM symbol interval.

Figure 4 shows the RMSE of the proposed normalized CFO estimator as a function of $E_{b} / N_{o}$ in AWGN and in multipath channels EVA and ETU. In the figure is also reported the derived CRB in (52). The results show that in AWGN channel the proposed estimator achieves the derived CRB for moderate and high values of $E_{b} / N_{o}$. A performance loss is observed in the considered multipath channels, however, the CFO estimate is quite accurate for $E_{b} / N_{o} \geq 5 \mathrm{~dB}$.

To gain some insight into the actual performance of the FBMC-PAM system when the proposed synchronization algorithm is exploited, in Fig.5 and 6 the BER of the perfectly synchronized FBMC-PAM-2M and FBMC-PAM-4M receivers in multipath channels EVA and ETU, respectively, is compared with that obtained in the case where the synchronization is performed with the proposed algorithm (curves labeled as FBMC-PAM-2M-s and FBMC-PAM-4M-s). In particular, in this first set of curves only the symbol timing is estimated while the CFO is assumed to be known. Moreover, in all the figures, the performance of the perfectly synchronized OFDM system with cyclic prefix $\mathrm{CP}=1 / 16$ in EVA channel and $\mathrm{CP}=1 / 8$ in ETU channel, is reported. The results show that in both channels the two considered receivers exploiting the proposed symbol timing estimation algorithm assure a performance indistinguishable from that achieved when the timing 
offset is perfectly known. Thus, also in the more frequency selective channel ETU, as previously observed, the RMSE of the symbol timing estimate is quite contained and, then, leads to a residual timing offset that the equalizer can handle.

The BER in multipath channels EVA and ETU of the FBMC-PAM-2M and FBMC-PAM-4M receivers when both the symbol timing and the CFO are estimated exploiting the proposed synchronization algorithm is reported in Fig.7 and 8, respectively. In the figures the labels FBMC-PAM-2M-s and FBMC-PAM-4M$\mathrm{s}$ refer to the case where the residual CFO is perfectly known and its effect (a phase shift constant on each subcarrier that linearly increases with the FBMCPAM symbol index) is perfectly balanced. Note that the residual CFO can be estimated in the frequency domain by exploiting pilot symbols inserted in the payload symbols of the burst. An efficient frequency domain CFO compensation algorithm for FBMC systems has been proposed in [13]. Moreover, in Fig.7 and 8 the labels FBMC-PAM-2M-nc and FBMC-PAM-4M-nc refer to the case where the residual $\mathrm{CFO}$ is not compensated. The results show that in both channels the two considered receivers exploiting the proposed synchronization algorithm assure a performance similar to that observed in the case of perfect synchronization (curves labeled as FBMC-APM-2M and FBMC-APM-4M), provided that the residual CFO is compensated in the frequency domain. As one would expect a performance degradation is observed in the absence of residual CFO compensation.

\section{Conclusions}

The FBMC-PAM transceiver with its capabilities in terms of spectral efficiency, asynchronous access and protection of adjacent users, has the potential to meet many requirements imposed by the future wireless systems. Here, the scenario in mind is short burst transmission which is a challenge for FBMC sys- 
tems because of the required length of preambles. With the proposed preamble, the initial loss of efficiency of FBMC-PAM with respect to the optimal 2M-OFDM approach is at most $2 \mathrm{M}$ real data. This loss can be more than compensated over the burst by the absence of the cyclic prefix.

In this paper, at first an analysis of the CFO sensitivity of the FBMC-PAM system is provided. In particular, an approximate expression of the SIR has been obtained and compared with the SIR of the OFDM system and of several offsetQAM multicarrier systems. The results have shown that the FBMC-PAM system is superior to the considered systems when all of them are subject to the same $\mathrm{CFO}$, that is when the total number of subcarriers of the FBMC-PAM system is equal to that of OFDM and of offset-QAM systems.

Then, the problem of preamble-based joint symbol timing and CFO estimation for FBMC-PAM transceivers has been considered. The derived symbol timing estimator requires a one-dimensional maximization while the CFO estimator is in closed form. Although the proposed algorithm is derived in AWGN channel, its performance is assessed in standard multipath channels. BER curves obtained via computer simulation have shown that when both the parameters are estimated the performance loss with respect to the ideal case is negligible for moderate and large values of $E_{b} / N_{o}$, provided that the residual CFO is accurately compensated in the frequency domain. 


\section{Appendix A}

In this appendix we obtain the expression of the $m$-th DFT value in (17). Since it depends also on the CFO we denote it as $R_{m}(i, \epsilon)$. Let us observe that, taking into account (3) in the absence of noise for $\theta=0$ and, without loss of generality, for $\gamma=1$, it follows that

$$
R_{m}(i, \epsilon)=\sum_{n=0}^{2 M-1} r[n+i M] e^{j \frac{2 \pi}{2 M} \epsilon(n+i M)} e^{-j \frac{2 \pi}{2 M} m n}=e^{j \pi \epsilon i}\left[A_{m}(i, \epsilon)+B_{m}(i, \epsilon)+C_{m}(i, \epsilon)\right]
$$

where

$$
\begin{gathered}
A_{m}(i, \epsilon) \triangleq \sum_{k=0}^{2 M-1}(-1)^{k} d_{k}[i-1] e^{j \frac{\pi}{M}\left(k+\frac{1}{2}\right)\left(\frac{1}{2}+\frac{M}{2}\right)} \sum_{n=0}^{M-1}\left(a-a^{*} e^{j \frac{\pi}{M} n}\right) e^{-j \frac{\pi}{M} n(m-\epsilon-k)}, \\
B_{m}(i, \epsilon) \triangleq \sum_{k=0}^{2 M-1} d_{k}[i] e^{j \frac{\pi}{M}\left(k+\frac{1}{2}\right)\left(\frac{1}{2}+\frac{M}{2}\right)} \sum_{n=0}^{2 M-1}\left(a+a^{*} e^{j \frac{\pi}{M} n}\right) e^{-j \frac{\pi}{M} n(m-\epsilon-k)},
\end{gathered}
$$

and

$$
C_{m}(i, \epsilon) \triangleq \sum_{k=0}^{2 M-1}(-1)^{k} d_{k}[i+1] e^{j \frac{\pi}{M}\left(k+\frac{1}{2}\right)\left(\frac{1}{2}+\frac{M}{2}\right)} \sum_{n=M}^{2 M-1}\left(a-a^{*} e^{j \frac{\pi}{M} n}\right) e^{-j \frac{\pi}{M} n(m-\epsilon-k)} .
$$

After simple but tedious algebra it follows that

$$
\begin{gathered}
e^{-j \frac{\pi}{M}\left(m+\frac{1}{2}\right)\left(\frac{1}{2}+\frac{M}{2}\right)}\left[a^{*} A_{m}(i, \epsilon)+a A_{m+1}(i, \epsilon)\right]= \\
\frac{1}{4} e^{j \frac{\pi}{2}} e^{j \frac{\pi}{2} \epsilon\left(1-\frac{1}{M}\right)}(-1)^{i} \sum_{k=0}^{2 M-1} d_{k}[i-1]\left\{\frac{\sin \left[\frac{\pi}{2}(m-\epsilon-k-1)\right]}{\sin \left[\frac{\pi}{2 M}(m-\epsilon-k-1)\right]}+\frac{\sin \left[\frac{\pi}{2}(m-\epsilon-k+1)\right]}{\sin \left[\frac{\pi}{2 M}(m-\epsilon-k+1)\right]}\right\}, \\
e^{-j \frac{\pi}{M}\left(m+\frac{1}{2}\right)\left(\frac{1}{2}+\frac{M}{2}\right)}\left[a^{*} C_{m}(i, \epsilon)+a C_{m+1}(i, \epsilon)\right]= \\
\frac{1}{4} e^{-j \frac{\pi}{2}} e^{j \frac{\pi}{2} \epsilon\left(3-\frac{1}{M}\right)} \sum_{k=0}^{2 M-1}(-1)^{k} d_{k}[i+1]\left\{\frac{\sin \left[\frac{\pi}{2}(m-\epsilon-k-1)\right]}{\sin \left[\frac{\pi}{2 M}(m-\epsilon-k-1)\right]}+\frac{\sin \left[\frac{\pi}{2}(m-\epsilon-k+1)\right]}{\sin \left[\frac{\pi}{2 M}(m-\epsilon-k+1)\right]},\right.
\end{gathered}
$$

and

$$
e^{-j \frac{\pi}{M}\left(m+\frac{1}{2}\right)\left(\frac{1}{2}+\frac{M}{2}\right)}\left[a^{*} B_{m}(i, \epsilon)+a B_{m+1}(i, \epsilon)\right]=\frac{1}{4} e^{j \frac{\pi}{2} \epsilon\left(2-\frac{1}{M}\right)} \sum_{k=0}^{2 M-1} e^{j \frac{3 \pi}{2}(k-m)} d_{k}[i]
$$




$$
\times\left\{2 \frac{\sin [\pi(m-\epsilon-k)]}{\sin \left[\frac{\pi}{2 M}(m-\epsilon-k)\right]}+\frac{\sin [\pi(m-\epsilon-k-1)]}{\sin \left[\frac{\pi}{2 M}(m-\epsilon-k-1)\right]}+\frac{\sin [\pi(m-\epsilon-k+1)]}{\sin \left[\frac{\pi}{2 M}(m-\epsilon-k+1)\right]}\right\} .
$$

Taking into account (A.1), by using (A.5), (A.6) and (A.7), the expression in (18) immediately follows.

\section{Appendix B}

In this appendix, we derive the approximate expressions of the power of the useful term in (21), the ISI contribution in (22), and the ICI term in (23), for $|\epsilon| \ll 1$ and $M \gg 1$.

Let us consider at first the power of the useful term. Note that, taking into account the definition in (19), for $M \gg 1$ and $|\epsilon| \ll 1$, it follows that

$$
2 D_{4 M}^{0}(2 \epsilon)+D_{4 M}^{-2}(2 \epsilon)+D_{4 M}^{2}(2 \epsilon) \simeq 4 M \frac{\sin (\pi \epsilon)}{\pi \epsilon} \frac{1}{1-\epsilon^{2}} \simeq 4 M .
$$

Thus, from (B.1) the expression in (24) is immediately obtained.

Let us now consider the power of the ISI contribution in (22). Note that, taking into account the definition in (19), it follows that

$$
D_{2 M}^{p+2 M}(\epsilon)=(-1)^{M} D_{2 M}^{p}(\epsilon)
$$

From the previous relationship it follows that

$$
\sum_{k=0}^{2 M-1}\left[D_{2 M}^{m-k-1}(\epsilon)\right]^{2}=\sum_{k=0}^{2 M-1}\left[D_{2 M}^{m-k+1}(\epsilon)\right]^{2}=\sum_{p=0}^{2 M-1}\left[D_{2 M}^{p}(\epsilon)\right]^{2}
$$

and, moreover,

$$
\sum_{k=0}^{2 M-1} D_{2 M}^{m-k-1}(\epsilon) D_{2 M}^{m-k+1}(\epsilon)=\sum_{p=0}^{2 M-1} D_{2 M}^{p-1}(\epsilon) D_{2 M}^{p+1}(\epsilon)
$$

Thus, the power of the ISI contribution in (22) can be expressed as

$$
P_{I S I}(\epsilon)=4\left[\sin \left(\frac{\pi}{2} \epsilon\right)\right]^{2}\left\{A_{I S I}+B_{I S I}\right\}
$$


where

$$
A_{I S I} \triangleq \sum_{p=0}^{2 M-1}\left[D_{2 M}^{p}(\epsilon)\right]^{2}
$$

and

$$
B_{I S I} \triangleq \sum_{p=0}^{2 M-1} D_{2 M}^{p-1}(\epsilon) D_{2 M}^{p+1}(\epsilon)
$$

In particular, taking into account the definition in (19), it follows that

$$
A_{I S I}=\left[\frac{\sin \left(\frac{\pi}{2} \epsilon\right)}{\sin \left(\frac{\pi}{2 M} \epsilon\right)}\right]^{2}+\sum_{p=1}^{2 M-1}\left[\frac{\sin \left(\frac{\pi}{2}(p-\epsilon)\right)}{\sin \left(\frac{\pi}{2 M}(p-\epsilon)\right)}\right]^{2} .
$$

For $M \gg 1$ and $|\epsilon| \ll 1$ it follows that

$$
\begin{gathered}
A_{I S I} \simeq M^{2}+\sum_{p=1}^{2 M-1}\left[\frac{\sin \left(\frac{\pi}{2} p\right)}{\sin \left(\frac{\pi}{2 M} p\right)}\right]^{2}=M^{2}+\sum_{l=0}^{M-1}\left[\frac{\sin \left(\frac{\pi}{2}(2 l+1)\right)}{\sin \left(\frac{\pi}{2 M}(2 l+1)\right)}\right]^{2} \\
=M^{2}+\sum_{l=0}^{M-1} \frac{1}{\left[\sin \left(\frac{\pi}{M}\left(l+\frac{1}{2}\right)\right)\right]^{2}} .
\end{gathered}
$$

Since it can be shown that (for $\lambda \neq m \pi$ )

$$
\sum_{k=0}^{M-1} \frac{1}{\left[\sin \left(\frac{\pi}{M} k+\frac{\lambda}{M}\right)\right]^{2}}=\frac{M^{2}}{\sin ^{2}(\lambda)}
$$

it results that

$$
A_{I S I} \simeq 2 M^{2} .
$$

Moreover, taking into account the definition in (19), it follows that

$$
B_{I S I}=\sum_{p=0}^{2 M-1} \frac{-1-\cos (\pi(p-\epsilon))}{\cos \left(\frac{\pi}{M}\right)-\cos \left(\frac{\pi}{M}(p-\epsilon)\right)} .
$$

For $M \gg 1$ and $|\epsilon| \ll 1$ it follows that

$$
B_{I S I} \simeq \frac{1+\cos (\pi \epsilon)}{\cos \left(\frac{\pi}{M} \epsilon\right)-\cos \left(\frac{\pi}{M}\right)}-\sum_{p=1}^{2 M-1} \frac{1+(-1)^{p}}{\cos \left(\frac{\pi}{M}\right)-\cos \left(\frac{\pi}{M} p\right)}
$$




$$
\begin{gathered}
\simeq \frac{4 M^{2}}{\pi^{2}}-\sum_{p=1}^{2 M-1} \frac{1+(-1)^{p}}{1-\cos \left(\frac{\pi}{M} p\right)}=\frac{4 M^{2}}{\pi^{2}}-\sum_{p=1}^{2 M-1} \frac{1+(-1)^{p}}{2\left[\sin \left(\frac{\pi}{2 M} p\right)\right]^{2}} \\
=\frac{4 M^{2}}{\pi^{2}}-\sum_{l=1}^{M-1} \frac{1}{\left[\sin \left(\frac{\pi}{M} l\right)\right]^{2}} .
\end{gathered}
$$

Since it can be shown that

$$
\sum_{k=1}^{M-1} \frac{1}{\left[\sin \left(\frac{\pi}{M} k\right)\right]^{2}}=\frac{M^{2}-1}{3}
$$

it results that

$$
B_{I S I} \simeq \frac{4 M^{2}}{\pi^{2}}-\frac{M^{2}-1}{3} \simeq M^{2}\left(\frac{4}{\pi^{2}}-\frac{1}{3}\right) .
$$

By substituting (B.11) and (B.15) in (B.5), the expression in (25) is obtained.

Let us finally consider the power of the ICI contribution in (23). Note that, taking into account the definition in (19) it follows that

$$
D_{4 M}^{2(p+2 M)}(2 \epsilon)=-D_{4 M}^{2 p}(2 \epsilon)
$$

From the previous relationship it follows that

$$
\begin{aligned}
& \sum_{k=0, k \neq m}^{2 M-1} \frac{\left[1+(-1)^{m-k}\right]}{2}\left[D_{4 M}^{2(m-k)}(2 \epsilon)\right]^{2}=\sum_{p=1}^{2 M-1} \frac{\left[1+(-1)^{p}\right]}{2}\left[D_{4 M}^{2 p}(2 \epsilon)\right]^{2} \\
& \sum_{k=0, k \neq m}^{2 M-1} \frac{\left[1+(-1)^{m-k}\right]}{2}\left[D_{4 M}^{2(m-k+1)}(2 \epsilon)\right]^{2}=\sum_{p=1}^{2 M-1} \frac{\left[1+(-1)^{p}\right]}{2}\left[D_{4 M}^{2 p+2}(2 \epsilon)\right]^{2} \\
& \sum_{k=0, k \neq m}^{2 M-1} \frac{\left[1+(-1)^{m-k}\right]}{2}\left[D_{4 M}^{2(m-k-1)}(2 \epsilon)\right]^{2}=\sum_{p=1}^{2 M-1} \frac{\left[1+(-1)^{p}\right]}{2}\left[D_{4 M}^{2 p-2}(2 \epsilon)\right]^{2}
\end{aligned}
$$

and, moreover,

$$
\sum_{k=0, k \neq 0}^{2 M-1} \frac{\left[1+(-1)^{m-k}\right]}{2} D_{4 M}^{2(m-k)}(2 \epsilon) D_{4 M}^{2(m-k+1)}(2 \epsilon)=\sum_{p=1}^{2 M-1} \frac{\left[1+(-1)^{p}\right]}{2} D_{4 M}^{2 p}(2 \epsilon) D_{4 M}^{2(p+1)}(2 \epsilon),
$$


$\sum_{k=0, k \neq 0}^{2 M-1} \frac{\left[1+(-1)^{m-k}\right]}{2} D_{4 M}^{2(m-k)}(2 \epsilon) D_{4 M}^{2(m-k-1)}(2 \epsilon)=\sum_{p=1}^{2 M-1} \frac{\left[1+(-1)^{p}\right]}{2} D_{4 M}^{2 p}(2 \epsilon) D_{4 M}^{2(p-1)}(2 \epsilon)$

$\sum_{k=0, k \neq 0}^{2 M-1} \frac{\left[1+(-1)^{m-k}\right]}{2} D_{4 M}^{2(m-k-1)}(2 \epsilon) D_{4 M}^{2(m-k+1)}(2 \epsilon)=\sum_{p=1}^{2 M-1} \frac{\left[1+(-1)^{p}\right]}{2} D_{4 M}^{2 p-1}(2 \epsilon) D_{4 M}^{2(p+1)}(2 \epsilon)$.

Thus, the power of the ICI contribution in (23) can be expressed as

$$
P_{I C I}(\epsilon)=4 A_{I C I}+B_{I C I}+C_{I C I}+4 D_{I C I}+4 E_{I C I}+F_{I C I}
$$

where

$$
\begin{gathered}
A_{I C I} \triangleq \sum_{p=1}^{2 M-1} \frac{\left[1+(-1)^{p}\right]}{2}\left[D_{4 M}^{2 p}(2 \epsilon)\right]^{2}=\sum_{l=1}^{M-1}\left[D_{4 M}^{4 l}(2 \epsilon)\right]^{2}, \\
B_{I C I} \triangleq \sum_{p=1}^{2 M-1} \frac{\left[1+(-1)^{p}\right]}{2}\left[D_{4 M}^{2 p+2}(2 \epsilon)\right]^{2}=\sum_{l=1}^{M-1}\left[D_{4 M}^{4 l+2}(2 \epsilon)\right]^{2}, \\
C_{I C I} \triangleq \sum_{p=1}^{2 M-1} \frac{\left[1+(-1)^{p}\right]}{2}\left[D_{4 M}^{2 p-2}(2 \epsilon)\right]^{2}=\sum_{l=1}^{M-1}\left[D_{4 M}^{4 l-2}(2 \epsilon)\right]^{2}, \\
D_{I C I} \triangleq \sum_{p=1}^{2 M-1} \frac{\left[1+(-1)^{p}\right]}{2} D_{4 M}^{2 p}(2 \epsilon) D_{4 M}^{2 p+2}(2 \epsilon)=\sum_{l=1}^{M-1} D_{4 M}^{4 l}(2 \epsilon) D_{4 M}^{4 l+2}(2 \epsilon), \\
E_{I C I} \triangleq \sum_{p=1}^{2 M-1} \frac{\left[1+(-1)^{p}\right]}{2} D_{4 M}^{2 p}(2 \epsilon) D_{4 M}^{2 p-2}(2 \epsilon)=\sum_{l=1}^{M-1} D_{4 M}^{4 l}(2 \epsilon) D_{4 M}^{4 l-2}(2 \epsilon),
\end{gathered}
$$

and, finally,

$$
F_{I C I} \triangleq \sum_{p=1}^{2 M-1} \frac{\left[1+(-1)^{p}\right]}{2} D_{4 M}^{2 p-2}(2 \epsilon) D_{4 M}^{2 p+2}(2 \epsilon)=\sum_{l=1}^{M-1} D_{4 M}^{4 l-2}(2 \epsilon) D_{4 M}^{4 l+2}(2 \epsilon)
$$

In particular, taking into account the definition in (19) it follows that

$$
A_{I C I}=\sum_{l=1}^{M-1}\left[\frac{\sin \left(\frac{\pi}{2}(4 l-2 \epsilon)\right)}{\sin \left(\frac{\pi}{4 M}(4 l-2 \epsilon)\right)}\right]^{2}
$$

For $M \gg 1$ and $|\epsilon| \ll 1$, and moreover, accounting for (B.14), it follows that

$$
A_{I C I} \simeq[\sin (\pi \epsilon)]^{2} \sum_{l=1}^{M-1} \frac{1}{\left[\sin \left(\frac{\pi}{M} l\right)\right]^{2}}=(\pi \epsilon)^{2} \frac{M^{2}-1}{3} .
$$


Taking into account the definition in (19) it follows that

$$
B_{I C I}=\sum_{l=1}^{M-1}\left[\frac{\sin \left(\frac{\pi}{2}(4 l+2-2 \epsilon)\right)}{\sin \left(\frac{\pi}{4 M}(4 l+2-2 \epsilon)\right)}\right]^{2} .
$$

For $M \gg 1$ and $|\epsilon| \ll 1$, and moreover, accounting for (B.10), it follows that

$$
B_{I C I} \simeq[\sin (\pi \epsilon)]^{2} \sum_{l=1}^{M-1} \frac{1}{\left[\sin \left(\frac{\pi}{M}\left(l+\frac{1}{2}\right)\right)\right]^{2}} \simeq(\pi \epsilon)^{2} M^{2}\left(1-\frac{4}{\pi^{2}}\right) .
$$

Taking into account the definition in (19) it follows that

$$
C_{I C I}=\sum_{l=1}^{M-1}\left[\frac{\sin \left(\frac{\pi}{2}(4 l-2-2 \epsilon)\right)}{\sin \left(\frac{\pi}{4 M}(4 l-2-2 \epsilon)\right)}\right]^{2} .
$$

For $M \gg 1$ and $|\epsilon| \ll 1$, and moreover, accounting for (B.10), it follows that

$$
C_{I C I} \simeq[\sin (\pi \epsilon)]^{2} \sum_{l=1}^{M-1} \frac{1}{\left[\sin \left(\frac{\pi}{M}\left(l-\frac{1}{2}\right)\right)\right]^{2}} \simeq(\pi \epsilon)^{2} M^{2}\left(1-\frac{4}{\pi^{2}}\right)=B_{I C I} .
$$

Taking into account the definition in (19) it follows that

$$
\begin{aligned}
D_{I C I} & =\sum_{l=1}^{M-1} \frac{\sin \left(\frac{\pi}{2}(4 l-2 \epsilon)\right)}{\sin \left(\frac{\pi}{4 M}(4 l-2 \epsilon)\right)} \frac{\sin \left(\frac{\pi}{2}(4 l+2-2 \epsilon)\right)}{\sin \left(\frac{\pi}{4 M}(4 l+2-2 \epsilon)\right)} \\
& =\sum_{l=1}^{M-1} \frac{\cos (2 \pi \epsilon)-1}{\cos \left(\frac{\pi}{2 M}\right)-\cos \left(\frac{\pi}{4 M}(8 l+2-4 \epsilon)\right)} .
\end{aligned}
$$

For $M \gg 1$ and $|\epsilon| \ll 1$, and moreover, accounting for (B.10), it follows that

$$
D_{I C I} \simeq-(\pi \epsilon)^{2} \sum_{l=1}^{M-1} \frac{1}{\left[\sin \left(\frac{\pi}{M}\left(l+\frac{1}{4}\right)\right)\right]^{2}} \simeq-(\pi \epsilon)^{2} M^{2}\left[2-\left(\frac{4}{\pi}\right)^{2}\right] .
$$

Taking into account the definition in (19) it follows that

$$
\begin{aligned}
E_{I C I} & =\sum_{l=1}^{M-1} \frac{\sin \left(\frac{\pi}{2}(4 l-2 \epsilon)\right)}{\sin \left(\frac{\pi}{4 M}(4 l-2 \epsilon)\right)} \frac{\sin \left(\frac{\pi}{2}(4 l-2-2 \epsilon)\right)}{\sin \left(\frac{\pi}{4 M}(4 l-2-2 \epsilon)\right)} \\
& =\sum_{l=1}^{M-1} \frac{\cos (2 \pi \epsilon)-1}{\cos \left(\frac{\pi}{2 M}\right)-\cos \left(\frac{\pi}{4 M}(8 l-2-4 \epsilon)\right)} .
\end{aligned}
$$


For $M \gg 1$ and $|\epsilon| \ll 1$, and moreover, accounting for (B.10), it follows that

$$
E_{I C I} \simeq-(\pi \epsilon)^{2} \sum_{l=1}^{M-1} \frac{1}{\left[\sin \left(\frac{\pi}{M}\left(l-\frac{1}{4}\right)\right)\right]^{2}} \simeq-(\pi \epsilon)^{2} M^{2}\left[2-\left(\frac{4}{\pi}\right)^{2}\right]=D_{I C I}
$$

Taking into account the definition in (19) it follows that

$$
\begin{gathered}
F_{I C I}=\sum_{l=1}^{M-1} \frac{\sin \left(\frac{\pi}{2}(4 l+2-2 \epsilon)\right)}{\sin \left(\frac{\pi}{4 M}(4 l+2-2 \epsilon)\right)} \frac{\sin \left(\frac{\pi}{2}(4 l-2-2 \epsilon)\right)}{\sin \left(\frac{\pi}{4 M}(4 l-2-2 \epsilon)\right)} \\
=\sum_{l=1}^{M-1} \frac{1-\cos (2 \pi \epsilon)}{\cos \left(\frac{\pi}{M}\right)-\cos \left(\frac{\pi}{4 M}(8 l-4 \epsilon)\right)} .
\end{gathered}
$$

For $M \gg 1$ and $|\epsilon| \ll 1$, and moreover, accounting for (B.14), it follows that

$$
F_{I C I} \simeq(\pi \epsilon)^{2} \sum_{l=1}^{M-1} \frac{1}{\left[\sin \left(\frac{\pi}{M} l\right)\right]^{2}} \simeq(\pi \epsilon)^{2} \frac{M^{2}-1}{3}=A_{I C I} .
$$

By substituting (B.31), (B.33), (B.35), (B.37), (B.39), and (B.41) in (B.23) the expression in (26) is obtained. 


\section{References}

[1] Y. Medjahdi, S. Traverso, R. Gerzaguet, H. Shaïek. R. Zayani, D. Demmer, R. Zakatia, J.P. Doré, M. Ben Mabrouk, D.Le Ruyet, Y.Louët and D. Roviras, On the road to 5G: comparative study of physical layer in MTC context, IEEE Access. vol. 5, pp. 26556-26581, 2017.

[2] D. Pinchon, P. Siohan, Derivation of analytical expressions for flexible PR low complexity FBMC systems, Proc. of 21th European Signal Processing Conference (EUSIPCO 2013), pp. 1-5, Sept. 2013.

[3] J. Nadal, C.A. Nour, A. Baghdadi, Design and evaluation of a novel short prototype filter for OFDM/OQAM modulation, IEEE Access. vol. 6, pp. 1961019625, March 2018.

[4] D. Mattera, M. Tanda, M. Bellanger, Filter bank multicarrier with PAM modulation for future wireless systems, Signal Processing 120 (2016) pp, 594-606.

[5] T. Fusco, A. Petrella, M. Tanda, Data-aided symbol timing and CFO synchronization for filter bank multicarrier systems, IEEE Trans. Wireless Commun. vol. 8, pp. 2705-2715, May 2009.

[6] D. Mattera, M. Tanda, Data-aided synchronization for OFDM-OQAM systems, Signal Processing 92 (2012) pp. 2284-2292.

[7] H. Saeedi-Sourck, S. Sadri, Y. Wu, B. Farhang-Boroujeny, Near maximumm likelihood synchronization for filter bank multicarrier systems, IEEE Trans. Wireless Commun. vol. 2, pp. 235-238, April 2013.

[8] H. Saeedi-Sourck, Y. Wu, J.W.M. Bergmans, S. Sadri, B. Farhang-oroujeny, Sensitivity anlysis of offset QAM multicarrier systems to residual carrier frequency and timing offsets, Signal Processing 91 (2011) pp, 1604-1612. 
[9] J. Zhang, X. Huang, "Autocorrelation based coarse timing with differential normalization", IEEE Trans. Wireless Commun. vol. 11, pp. 526-530, Feb. 2012.

[10] M. Morelli, U. Mengali "An improved frequency offset estimator for OFDM applications", IEEE Communications Letters, vol.3, no.3, pp. 75-77, March 1999.

[11] H. Minn, V.K. Bhargava, K.B. Letaief, "A robust timing and frequency synchronization for OFDM systems", IEEE Transactions on Wireless Communications, vol.2, no.4, pp. 822-839, July 2003.

[12] 3GPP TS 136104 version 8.8.0 Release 8, 2010.

[13] D. Mattera, M. Tanda, M. Bellanger, Frequency domain CFO compensation for FBMC systems, Signal Processing 114 (2015) pp, 183-197. 


\section{List of figures}

Fig. 1 SIR as a function of the normalized CFO.

Fig. 2 Behaviour of the proposed symbol timing statistics as a function of $\tilde{\theta}$ in a single run for $M=512$ and $E_{b} / N_{o}=10 \mathrm{~dB}$.

Fig. 3 Normalized RMSE of the proposed symbol timing estimator over AWGN, EVA and ETU channels versus $E_{b} / N_{o}$.

Fig. 4 RMSE of the proposed CFO estimator over AWGN, EVA and ETU channels versus $E_{b} / N_{o}$.

Fig. 5 BER versus $E_{b} / N_{o}$ over EVA channel. The proposed symbol timing estimator is exploited while the CFO is assumed to be known.

Fig. 6 BER versus $E_{b} / N_{o}$ over ETU channel. The proposed symbol timing estimator is exploited while the CFO is assumed to be known.

Fig. 7 BER versus $E_{b} / N_{o}$ over EVA channel. Both the symbol timing and the CFO are estimated.

Fig. 8 BER versus $E_{b} / N_{o}$ over ETU channel. Both the symbol timing and the CFO are estimated. 


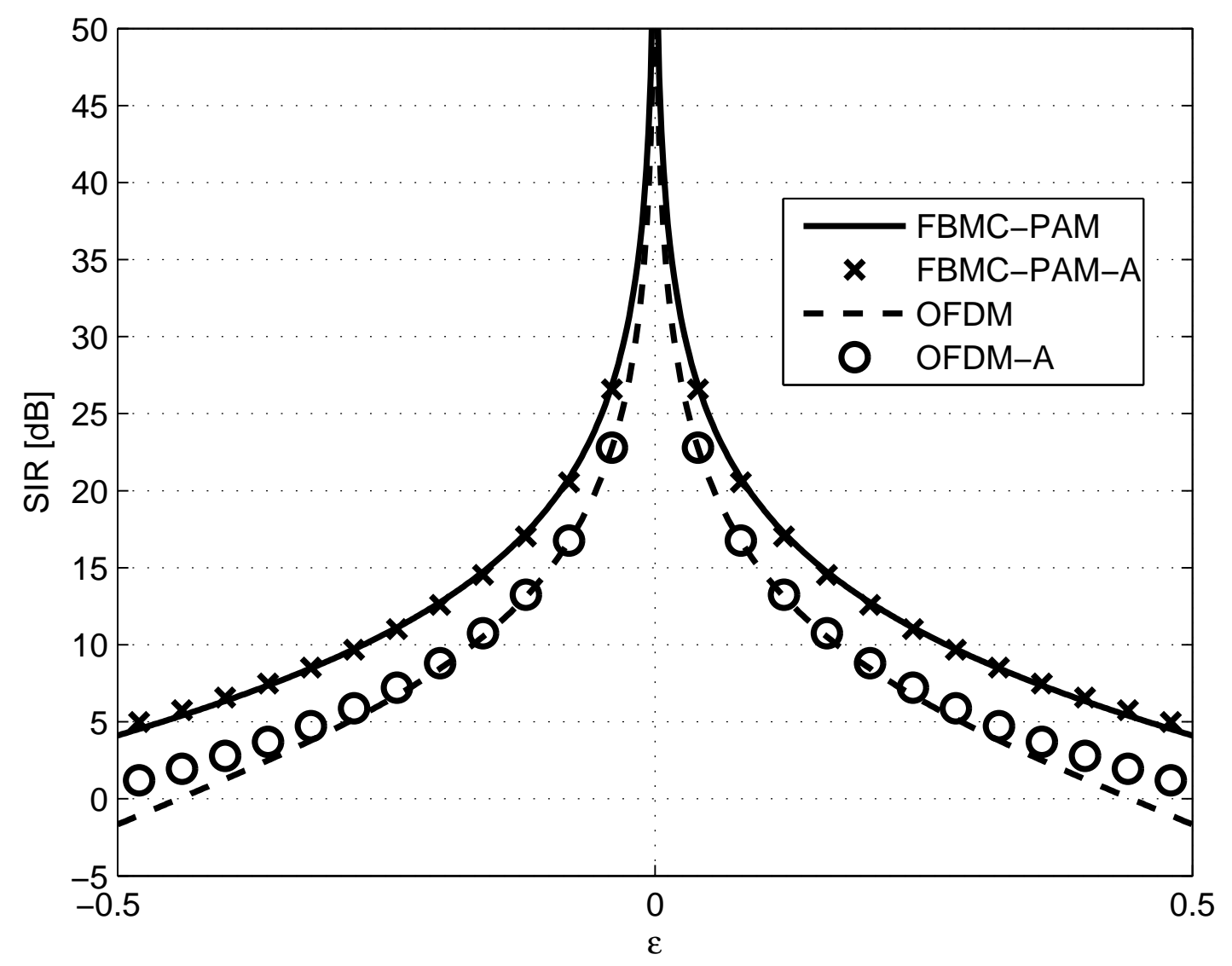

Figure 1: SIR as a function of the normalized CFO. 


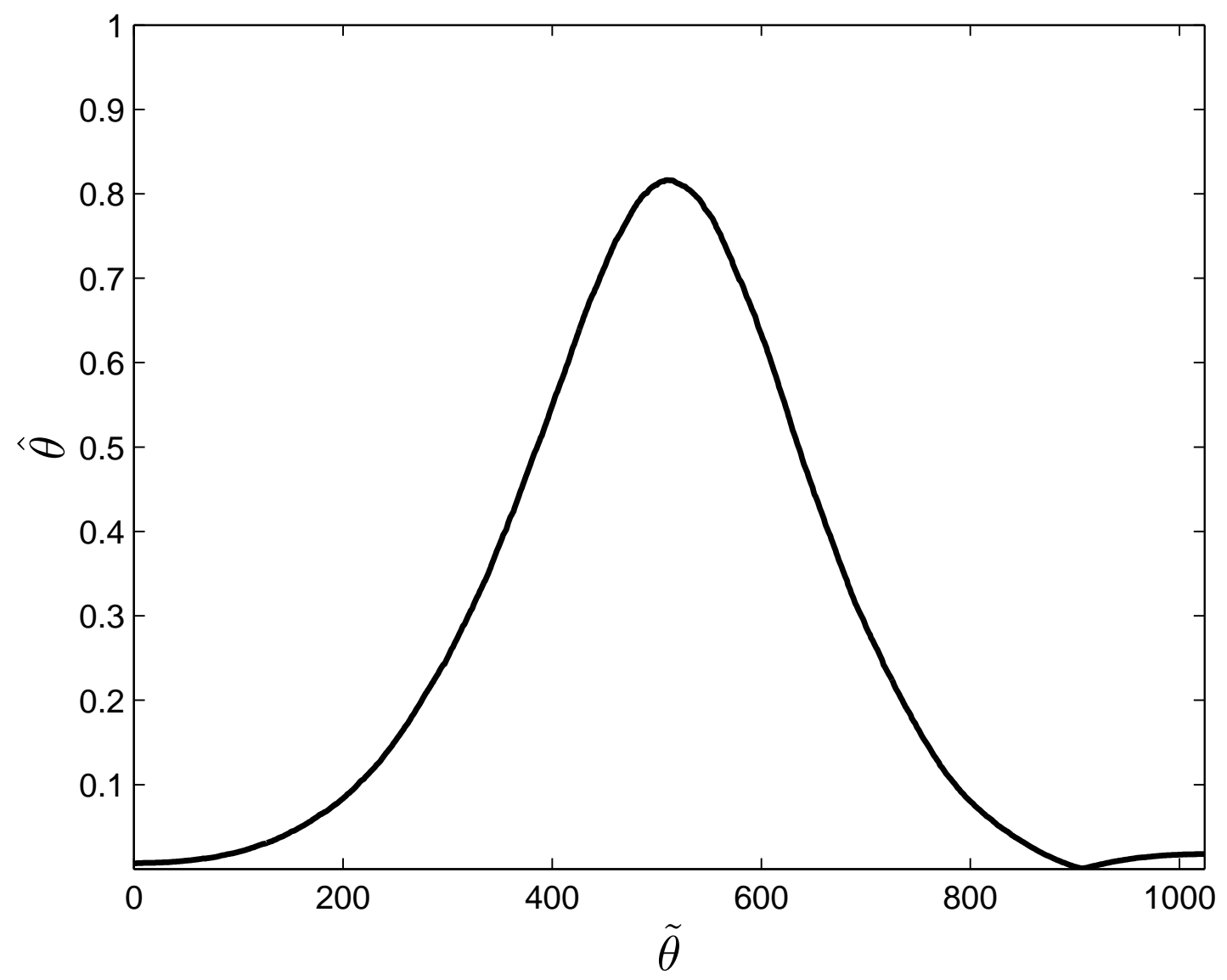

Figure 2: Behaviour of the proposed symbol timing statistics as a function of $\tilde{\theta}$ in a single run for $M=512$ and $E_{b} / N_{o}=10 \mathrm{~dB}$. 


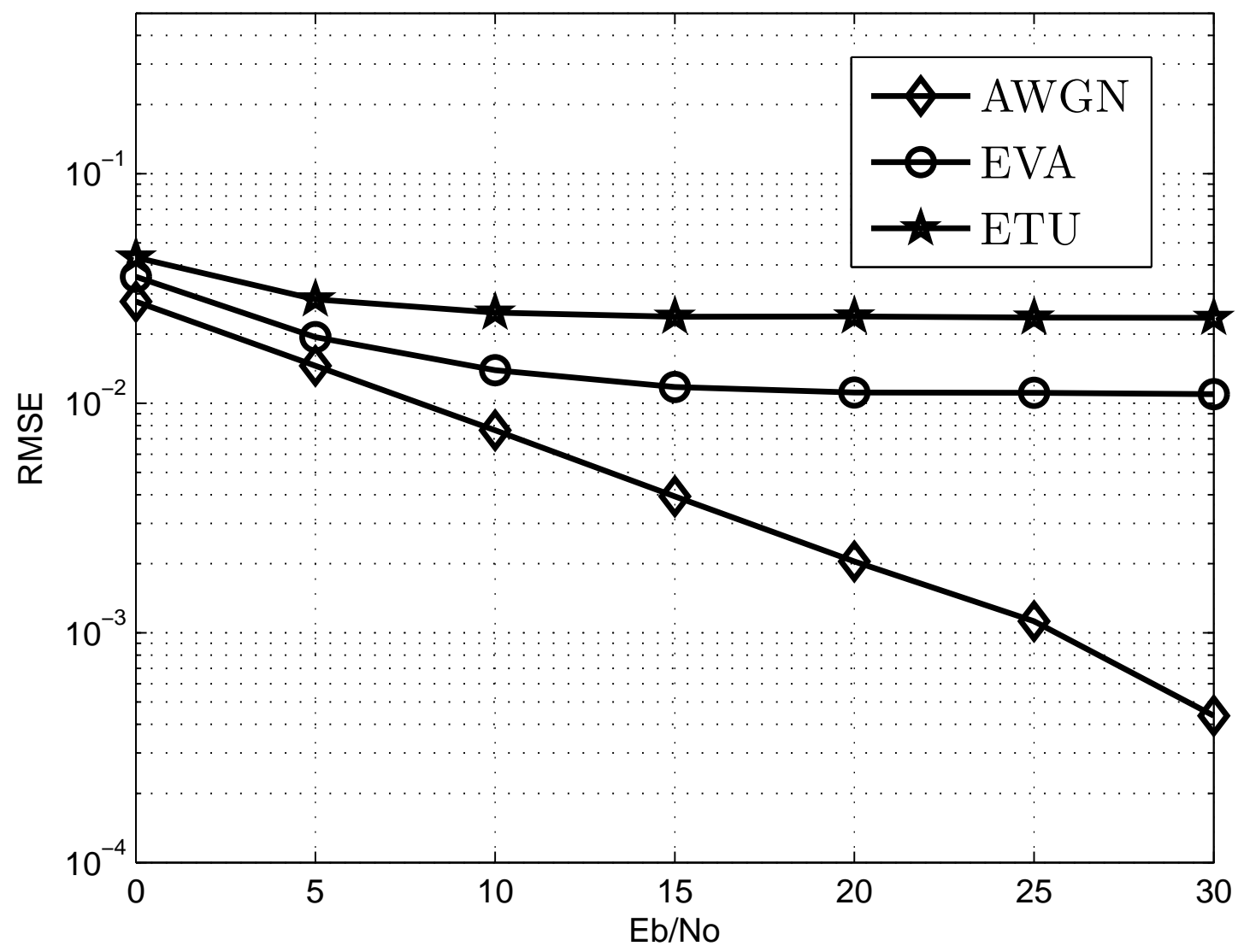

Figure 3: Normalized RMSE of the proposed symbol timing estimator over AWGN, EVA and ETU channels versus $E_{b} / N_{o}$. 


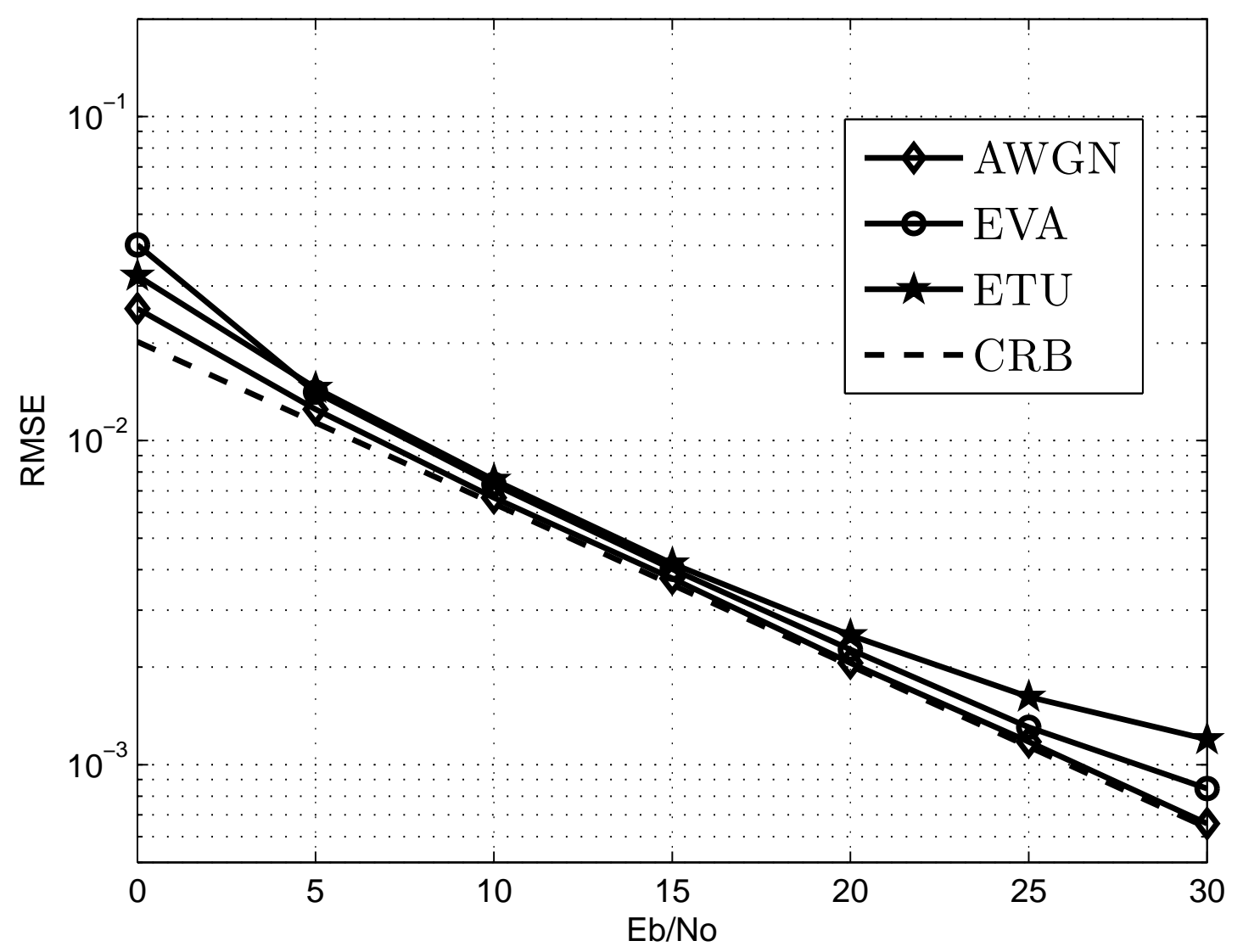

Figure 4: Normalized RMSE of the proposed CFO estimator over AWGN, EVA and ETU channels versus $E_{b} / N_{o}$. 


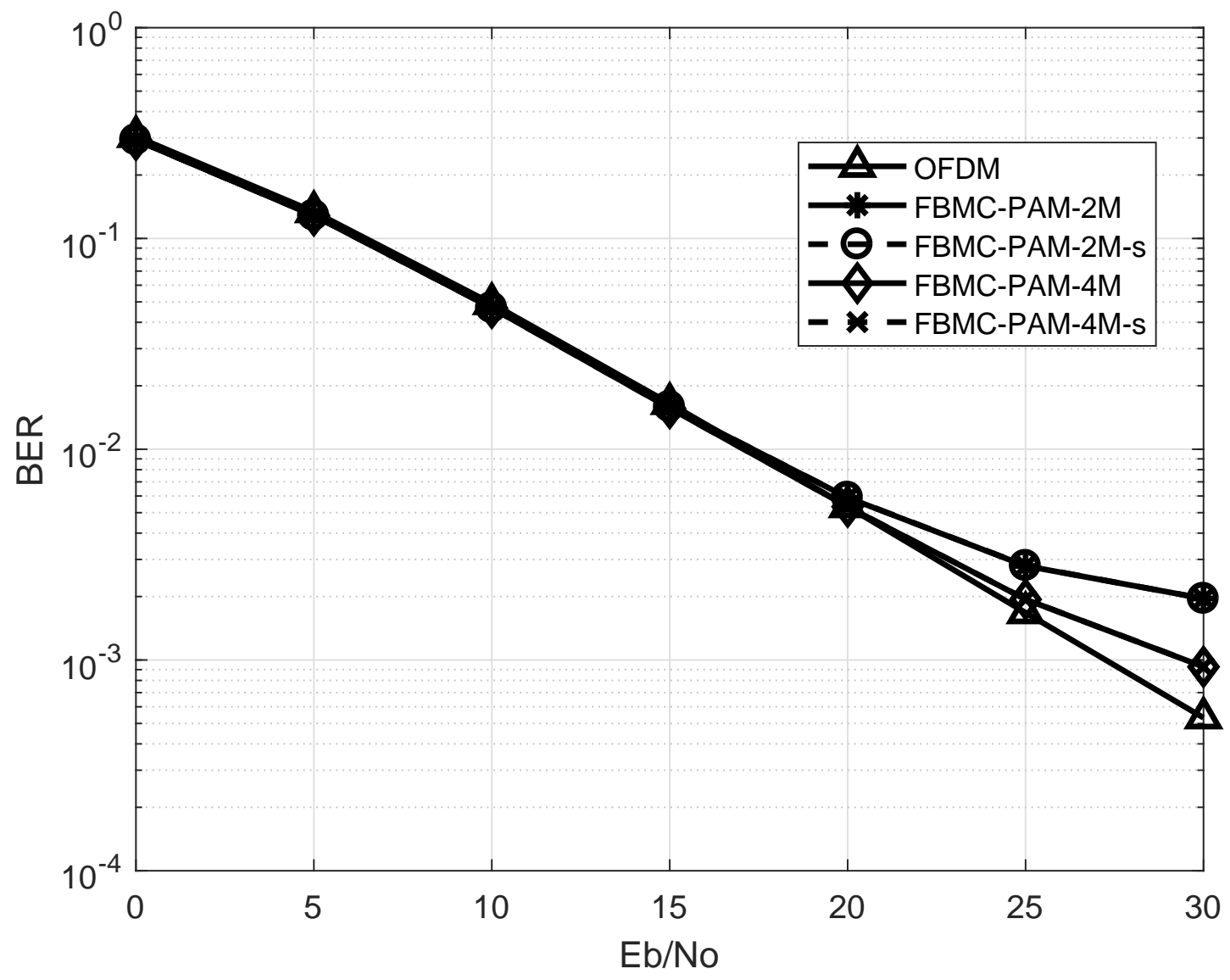

Figure 5: BER versus $E_{b} / N_{o}$ over EVA channel. The proposed symbol timing estimator is exploited while the $\mathrm{CFO}$ is assumed to be known. 


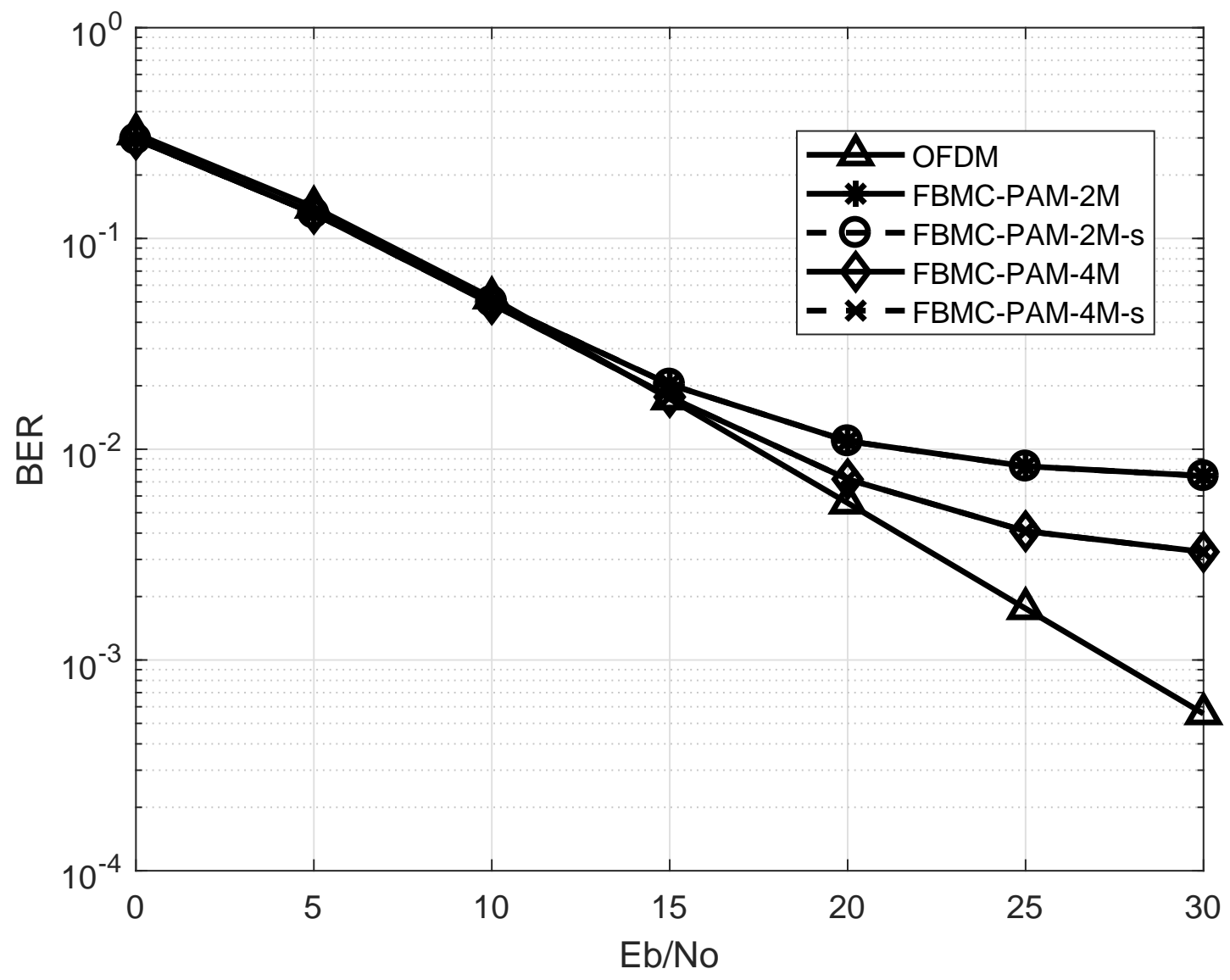

Figure 6: BER versus $E_{b} / N_{o}$ over ETU channel. The proposed symbol timing estimator is exploited while the $\mathrm{CFO}$ is assumed to be known. 


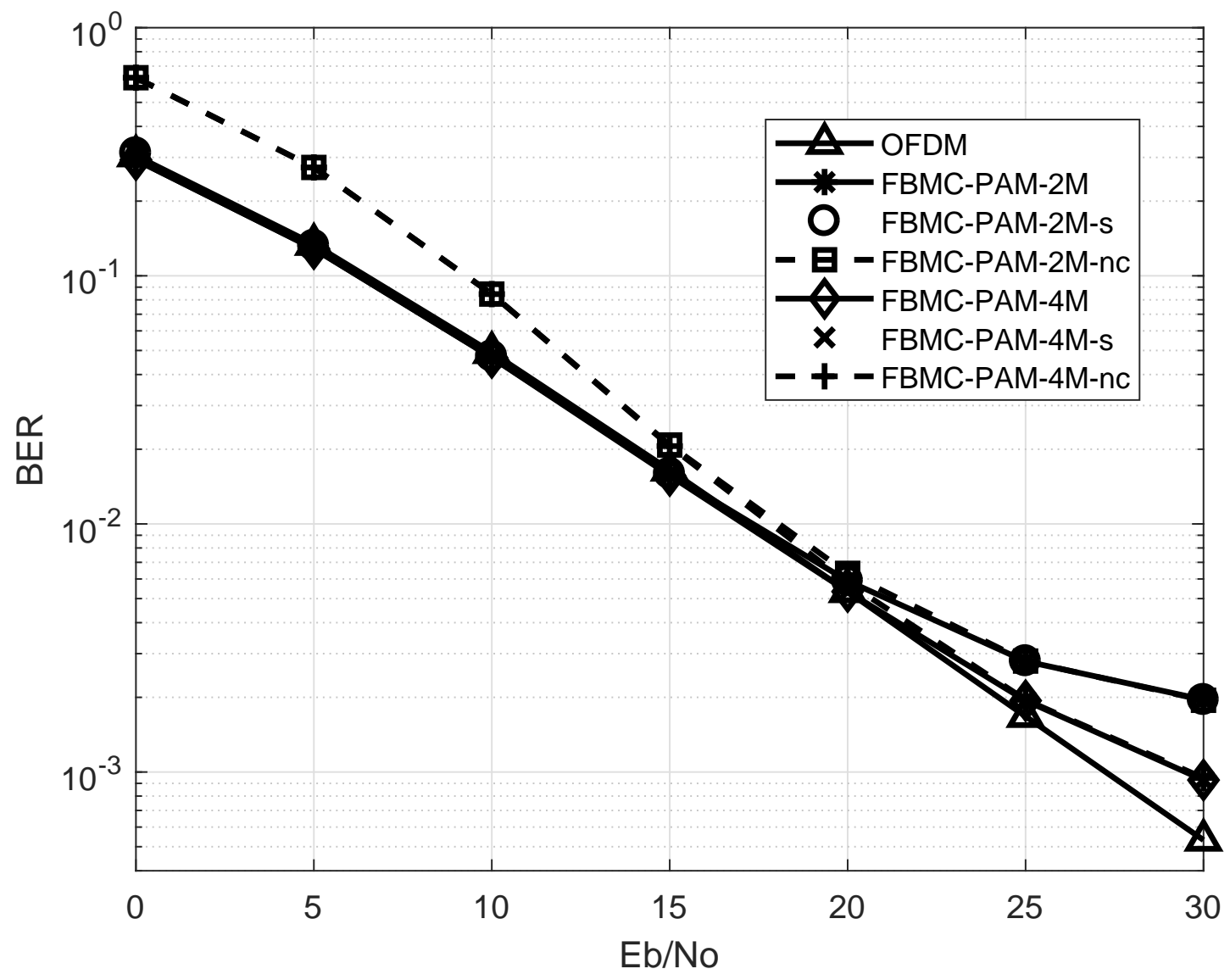

Figure 7: BER versus $E_{b} / N_{o}$ over EVA channel. Both the symbol timing and the CFO are estimated. 


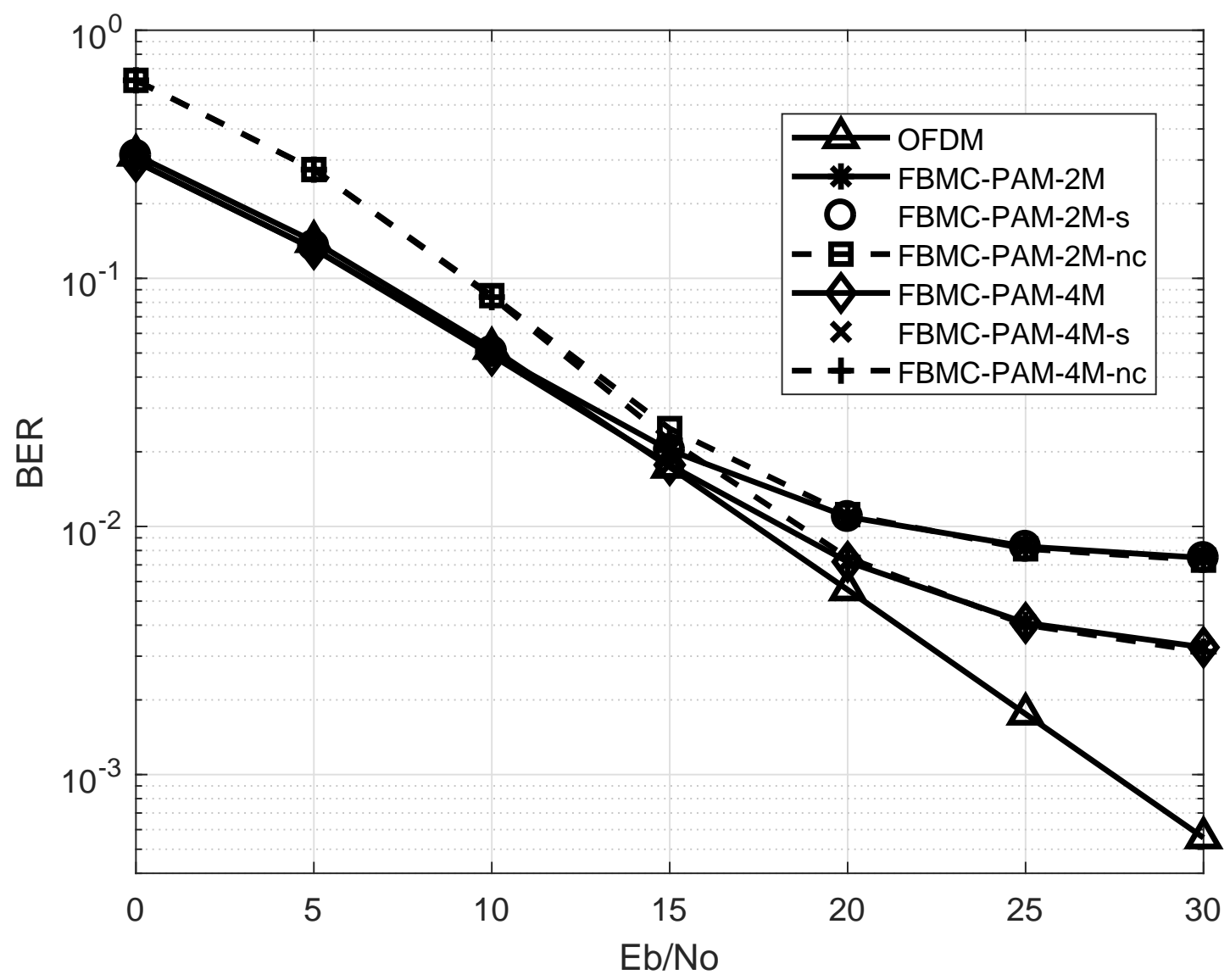

Figure 8: BER versus $E_{b} / N_{o}$ over ETU channel. Both the symbol timing and the CFO are estimated. 